\title{
Tumor de Wilms en la infancia
}

DRS. VICTORLA BERESI *, ERIKA QUINTANA **, JUAN JOSE LATORRE ***, ALFREDO DEL RIO ****, GASTON RIVERA * Y HUMBERTO DEL POZO*.

InTRoDucción. El nefroblastoma o Tumor de Wilms es un tumor maligno renal derivado de restos del mesonefro embrionario.

Este tumor en estadísticas extranjeras constituye un 20 a $30 \%$ de todos los tumores malignos de la infancia (10) (18) y un 6\% de todos los tumores renales. Estas cifras no están de acuerdo con nuestra realidad nacional ( 7 ) (30) en que el Wilras corresponde a un $10 \%$ de la totalidad de los tumores. En nuestra casuística de 153 tumores, 16 corresponden a nefroblastoma, lo que da un porcentaje de $9,8 \%$.

Se ha planteado la posibilidad de que el Wilms tenga un origen pre-natal basado en: aspecto bistológico embrionario, mayor incidencia en el niño pequeño, presencia de este tumor en fetos y recién nacidos (Tumor congénito) y su ocasional asociación con malformaciones congénitas (aniridia, hemihipertrofia, hamartomas, anomalías génito-urinarias, etc.) (5) (12) (14) (17) 20) (25) (33). Estas observaciones sugieren que el tumor podría ser inducido por un agente que tendría efecto oncogénico y teratogénico a la vez (12) (25).

En trabajos recientemente publicados parece ser que el pronóstico hasta hace poco tan sombrío en este tipo de tumores, puede ser mejorado gracias a un conocimiento más acabado de su sintomatología y una juiciosa aplicación de nuevas modalidades terapéuticas (10) (18) (29).

Material y MÉtodo. Se analizan todos los pacientes con tumor de Wilms que fueron atendidos en el Centro de Tumores del Hospital Luis Calvo Mackenna durante el período comprendido

\footnotetext{
- Centro Oncología, Hospital Luis Calvo Mackenna.

* Departamento de Pedíatra, Hospital L. Calvo Mackenna.

** Departamento Anatomia Patológica, Hospital L. Calvo Mac-

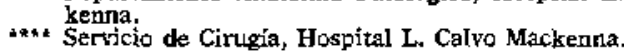

entre Diciembre de 1967 al 30 de Junio de 1972 , y que corresponden a 15 casos.

El ingreso de un niño con tumor abdominal plantea una situación de urgencia médico-quirúrgica que debe ser resuelta en un plazo no mayor de 48 hrs. En este tiempo debe realizarse un estudio lo más completo posible, cuyos principales objetivos sean: diaguóstico precoz, localización del tumor y prevención de la diséminación.

En nuestros pacientes procedemos en el orden siguiente:

\section{- Anamnesis.}

- Examen físico completo, cuidadosa palpación del tumor a fin de determinar su tamaño, pero evitando la manipulación excesiva e indiscriminada que puede ocasionar diseminación de células neoplásicas o ruptura del tumor.

- Exámenes de laboratorio: velocidad de eritrosedimentación, Hemograma, Haptoglobina, Orina, Uremia.

- Estudio radiológico: Radiografía de Tórax, de Abdomen simple y Pielografía.

Una vez completado el estudio se procede a Nefrectomía en el plazo anteriormente establecido, a menos que existan metástasis extra-abdominales, bilateralidad del tumor o excesivo volumen de éste.

La nefrectomía se realiza por vía transabdominal con incisión amplia que permita un buen campo operatorio. Ligadura del pedículo vascular renal antes de jniciar la manipulación y resección del tumor. Se procede además a la búsqueda sistemática de compromișo de cápsula renal y estructuras vecinas: vasos, sistema pielocaliciario, ganglios linfáticos y compromiso de vísceras abdominales (bazo, hígado, intestino, riñón opuesto). 
Se practica estudio histopatológico del tumor, de ganglios resecados y de muestras de tejidos aparentemente comprometidos o sospcchosos de infiltración neoplásica.

Una vez concluido este estudio, que exige colaboración del clínico, cirujano y anátomopato logo, es posible deducir una clasificación do estadio clínico que es importante para decidir tratamiento y pronóstico (10) (26). (tabla No 1 ).

T A B L A No 1

CLASIFICACION CLINICA DEL T. DE WILMS *

ESTADIO I.- T. QUE COMPROMETE SOLO EL RINOON.

ESTADIO II- T. QUE COMPROMETE LA FOSA RENAL.

a) Invasión de cápsula renal, pero no visceras adyacentes o metástasis.

b) Invasión de vasos renales.

c) Invasión de vasos linfáticos del hilio renal.

ESTADIO III.- T. QUE COMPROMETE EL ABDOMEN.

a) Invasión de viscera adyacente por extensión directa.

b) Metástasis dentro del abdomen. Ruptura de tumor. T. primario no extirpable.

c) T. Wilms bilateral, Invasión de vena cava.

ESTADIO IV.- METASTASIS FUERA DEL ABDOMEN : PULMONES, CERERRO $Y$ HUESO.

* Debe realizarse por cirtujano y anatomapatólogo,

El tratamiento de T. de Wilms además de la nefrectomía, lo constituye la Radioterapia y Quimioterapia.

En los casos de nefroblastoma unilateral y extirpable, en el momento del acto quirúrgico se inicia tratamiento con Actinomjcina $D$ en dosis de 15 gamas por $\mathrm{Kg}$. de peso, cura de 5 días, la que se repite al mes y medio, a los 3 meses y después cada 3 meses hasta completar 8 curas (1er. Esquema).

Después de la intervención y lo más pronto posible se inicia tanbién radioterapia, practicada en nuestros casos en la Fundación Arturo López Pérez.

Desde mayo del año 1972 utilizamos el esquema de tratamiento de Fleming (10) basado en estadios del tumor de Wilms (20 Esquema) (tabla 2).

A continuación de la primera cura el paciente debe ser controlado en forma periódica tanto del punto de vista médico, desarrollo psicomotor, curya
TA B L A NO 2

TRATAMIENTO BASADO EN ESTADIOS DE T. WILMS

\begin{tabular}{|c|c|c|c|}
\hline Estadio & Cirugia & Radiactón & Quimioterapia \\
\hline $\mathbf{I}$ & \multicolumn{2}{|c|}{$\begin{array}{c}\text { Nefrectomía } 2000-3000 \text { Rads en } \\
\text { fosa realal }\end{array}$} & $\begin{array}{l}\text { Ninguna en }<1 \text { año } \\
\text { Vinc }+ \text { Act, Do Sem. }\end{array}$ \\
\hline II A y $\mathbf{B}$ & $n$ & $\begin{array}{l}200(-33) \text { Rads } 50- \\
\text { bre fosa renal }+ \\
2 \text { cms, margen. }\end{array}$ & $\begin{array}{l}\text { Vinc + Act D } 2 \text { curas } \\
6 \text { meses. }\end{array}$ \\
\hline II C & $"$ & $\begin{array}{l}2000-3500 \text { Rads so- } \\
\text { bre todo abdomen } \\
\text { evitando riñón } \\
\text { opuesto y pelvis } \\
\text { femenina. }\end{array}$ & $\begin{array}{l}\text { Vinc + Act. D. } 4 \text { curas } \\
12 \text { meses. }\end{array}$ \\
\hline III & $\begin{array}{l}\text { R. cuando } \\
\text { es posible }\end{array}$ & $\begin{array}{l}\text { 1.000-3.000 } \\
\text { Rads sobre todo } \\
\text { abdomen evitan- } \\
\text { do ringn opuesto. }\end{array}$ & $\begin{array}{l}\text { Vinc + Act. D } 4 \text { curas } \\
12 \text { meses: rcevaluación. }\end{array}$ \\
\hline IV & $\begin{array}{l}\text { N. cuando } \\
\text { cs posib]e }\end{array}$ & $\begin{array}{l}\text { o Segín caso parti- } \\
\text { cular (tarnaño de } \\
\text { campos y dosis). }\end{array}$ & $\begin{array}{l}\text { Vine + Act. D } 4 \text { muras } \\
12 \text { meses; reevaluación. }\end{array}$ \\
\hline
\end{tabular}

pondoestatural, como también mediante exámenes de laboratorio: Velocidad de eritrosedimentación, Haptoglobina, Hemograma y Radiografía de Tórax cada mes y medio durante 18 meses. Después de este plazo los controles se realizan cada 3 ó 6 meses.

Durante el tratamiento quimioterápico se controla estrechamente el recuento de blancos, y si esta cifra cae a menos de 2.000 por $\mathrm{mm}^{3}$, se suspende transitoriamente.

La radiografía de Tórax periódica tiene por objeto pesquisar precozmente las metástasis pulmonares que en general son de evolución asintomática,

\section{DISTRIBUCION POR SEXO}

\section{$N^{*} \operatorname{cosos}$}

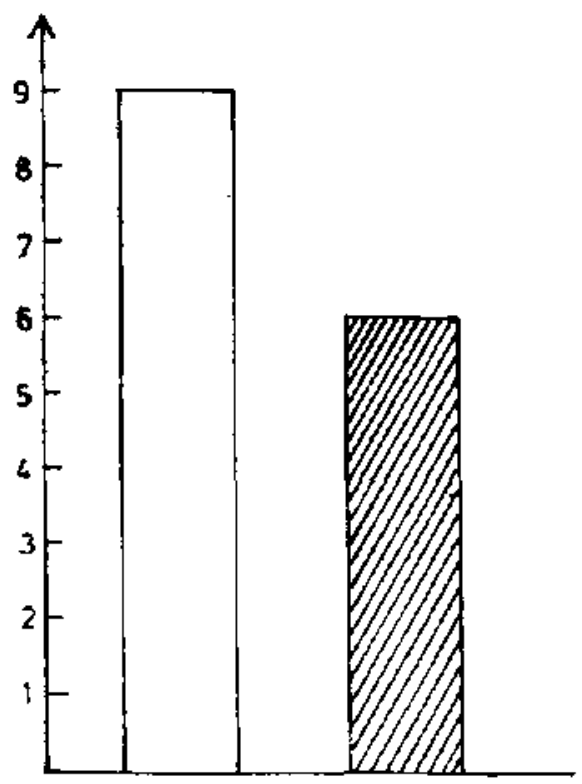

Figura Ni: 1 
REsultados y comentariós. En nuestra serie no se aprecian diferencias significativas en cuanto a sexo, lo que está de acuerdo con publicaciones tanto nacionales como extranjeras (7) (18) (30). (Figura No 1).

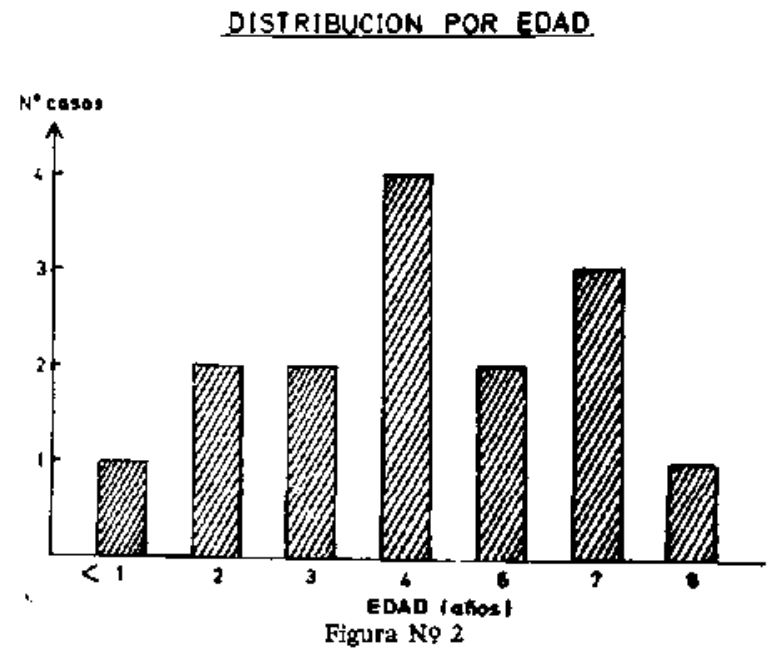

En la figura № 2 se observa una marcada incidencia en los menores de 7 años, y especialmente en el menor de 4 años, hecho que se repite en la literatura (1) 18) (20 (24) en la cual se reconoce que el Nefroblastoma es un tumor del niño menor de 7 años y que un $70 \%$ aparece antes de los 4 años (18).

TA BLA No 3

PERIODO PRE-DIAGNOSTICO

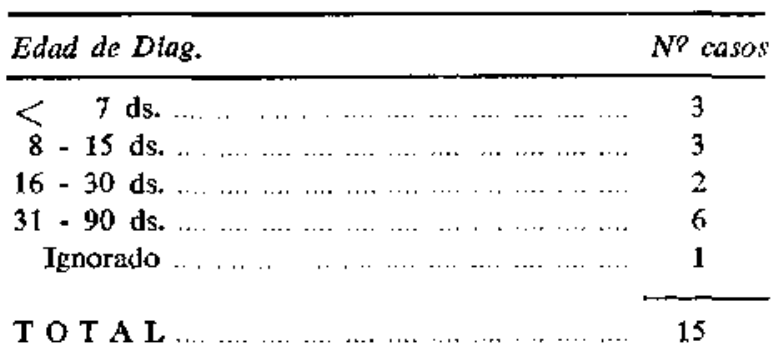

En la tabla No 3 analizamos el tiempo transcurrido entre el comienzo de los síntomas y el diagnóstico de la enfermedad, y vemos que sólo en 6 de nuestros pacientes éste se hizo antes de los 15 días, es decir, en un plazo que nos parece aceptable.

En 8 casos fue tardio debido a que presentaro nmanifestaciones vagas y diversas, hecho por lo demás bastante característico en este tipo de tumor.

Para algunos autores (18) el período de invasión tumoral, que es el tiempo que transcurre entre la aparición de las primeras manifestaciones clínicas y el momento del diagnóstico, es de 2

a 3 meses lo que incide en su mal pronostico. De esto se deduce la importancia del conocimiento clínico de la enfermedad y la interpretación de síntomas que pueden ser banales e intermitentes. SINIOMAS Y SIGNOS

DOLOR ABDOMINAL

ENFLAQUECIMIENTO

TUMOR

YOMITOS

FIEBRE

PALIDEZ

ABDONEN AGUDO

CONSTIPACKON

DECAIMIENTO

DISNEA

DIARREA

HEMATURIA

ANOREXIA
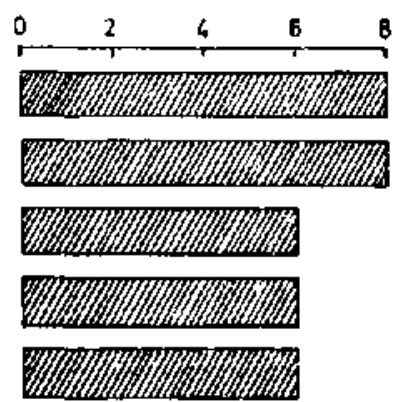

EPISTAXIS

HIPERTENSION ARTERIAL WIIA

Figura No 3

En nuestro material los síntomas predominantes fueron dolor abdominal y enflaquecimiento, le siguen en frecuencia, tumor palpable, vómitos, fiebre y palidez. Menos frecuentes fueron: signos de abdomen agudo, constipación, decaimiento, disnea, diarrea, anorexia, epistaxis, hematuria macroscópica e hipertensión arterial (Figura No 3).

Lo habitual en el nefroblastoma es el desarroIlo insidioso e indoloro (24) (25), hasta que el tumor es evidente, las manifestaciones digestivas, urinarias y generales son tardias.

T A B L A No 4

\section{CARACTERISTICAS DEL TUMOR}

Localización:Flanco y fosa lumbar

Tamaño: $\quad 8-22 \mathrm{cms}$. No sobrepasa linea media

Ubicación Sobrepasa línea media
Der. 8 casos Izq. 7 casos 15 casos 14 casos 1 caso 
Entre los hallazgos del examen físico to esencial fue la palpación de un tumor abdominal descubierto en forma accidental o por examen sistemático.

Generalmente el tumor es ovalado, de superficie irregular, de consistencia firme, indoloro y fijo. De tamaño variable, de ubicación preferentemente abdominal más que lumbar (1) (7) (18) (23) (25).

En nuestros casos no hubo localización preferencial ni bilateralidad. Hay autores que dan un $2 \%$ de predominio izquierdo (17) y 10 (12) a $12 \%$ (7) de tumor bilateral.

En lo que se refiere a tamaño, en todos los pacientes fue de un volumen importante que fluctuó entre 8 y $22 \mathrm{~cm}$. ubicado en flanco y fosa lumbar no sobrepasando la línea media salvo en un caso y que explicamos por tamaño excesivo del tumor. A éste último hecho se le asigna valor en diagnóstico diferencial con Neuroblastoma.

METASTASIS DE INGRESO

$N^{*}$ de CASOS

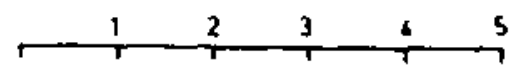

PULMONAR

SUPRARRENAL

HEPATICA

INTESTINAL
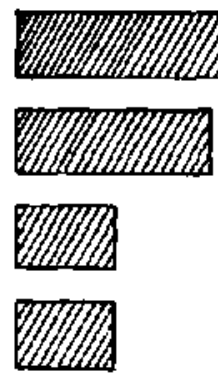

Figura No 4

Las metástasis del T. de Wilms se hacen generalmente por extensión directa en $80 \%$ (25) de los casos, también por diseminación hematógena (1) (7) (10) (25) (invasión de vena renal o cava) y por diseminación linfática (22) (25).

Los sitios de predilección son en orden de frecuencia: pulmones, hígado, riñón opuesto, cerebro, y más raro, metástasis ósea (7) (23) (25).

En nuestros pacientes con metástasis de ingreso la mayoría corresponde a localización pulmonar, múltiple en 4 casos y única en 1 . Los ofros órganos comprometidos fueron: suprarrenal, hígado $e$ intestino. No hemos encontrado tumor bilateral ni otro tipo de metástasis.

La velocidad de eritrosedimentación y haptoglobina, son un buen índice de control de nuestros niños, ya que en la mayoría de los casos que evolucionaron mal, permanecieron elevadas.

Pielografía se practicó en todos los casos pero en 4 desconocemos su resultado porque fue realizada en otras ciudades del país. De los 11 casos
T A B I A № 5

EXAMENES DE LABORATORIO

\begin{tabular}{|c|c|c|c|}
\hline \multicolumn{3}{|c|}{ Ingreso } & Evolución \\
\hline $\begin{array}{l}\text { Velocidad } \\
\text { eritrosediment. }\end{array}$ & $\left\{\begin{array}{c}N \\
\uparrow \\
\text { Desconocida }\end{array}\right.$ & $\begin{array}{l}2 \text { casos } \\
12 \text { casos } \\
1 \text { caso }\end{array}$ & $\begin{array}{l}\text { N } 6 \text { casos } \\
\text { t. } 9 \text { casos }\end{array}$ \\
\hline Orina hematuria & $(-)$ & & $(-)$ \\
\hline Haptoglobina & $\left\{\begin{array}{c}N \\
\text { No efectuada }\end{array}\right.$ & $\begin{array}{l}2 \text { casos } \\
6 \text { casos } \\
7 \text { casos }\end{array}$ & $\begin{array}{l}\text { N } 7 \text { casos } \\
+3 \text { casos }\end{array}$ \\
\hline Pielografía & $\begin{array}{l}\text { Exclusión renal } \\
\text { Distorsión y desplazamien } \\
\text { to sist. Pielo-caliciario } \\
\text { Desconocida }\end{array}$ & $\begin{array}{l}3 \text { casos } \\
8 \text { casos } \\
4 \text { casos }\end{array}$ & \\
\hline $\begin{array}{l}\text { Radlografia } \\
\text { de tórax }\end{array}$ & $\begin{array}{l}\text { Normal } \\
\text { Metástasis pulmonar } \\
\text { única } \\
\text { Metástasis mútíples }\end{array}$ & $\begin{array}{l}10 \text { casos } \\
1 \text { caso } \\
4 \text { casos }\end{array}$ & \\
\hline
\end{tabular}

restantes, 3 de ellos presentaron exclusion renal debida al gran tamaño del tumor. La distorsión y desplazamiento del sistema pielocaliciario ( $8 \mathrm{ca}$ sos) es lo característico del Nefroblastoma, a diferencja del Neuroblastrima que produce rechazo pero no invasión de él (1) (23).

TABLA No 6

ANATOMIA PATOLOGICA

PESO TUMOR

$<500$

4 casos

$500-1.000$ grs.

$>1.000$ grs.

Desconocido

1

$4 \pi$

Derecho

LOCALIZACION Izquierdo

Bilateral

$6 n$

COMPROMISO

$\begin{array}{lll}\text { Vasos } & 4 & " \\ \text { Cápsula renal } & 4 & " \\ \text { Suprarrenal } & 2 & " \\ \text { Ganglios } & 2 & " \\ \text { Sist. pielocaliciario } & 2 & " \\ \text { Hígado } & 2 & " \\ \text { Intestino } & 1 & \text { " } \\ \text { Bazo } & 1 & \end{array}$

Macroscópicamente estas neoplasias son voluminosas y con pesos que fluctúan entre $200 \mathrm{y}$ 2.000 grs. En la literatura se describen tumores de hasta $13 \mathrm{Kg}$. de peso. (Tabla No 6 ).

Al corte revelan un aspecto moteado según sea el tipo tisular. Areas mixomatosas, blandas, de color gris. A veces se reconocen estructuras cartilaginosas hialinas y necrosis hemorrágicas. 


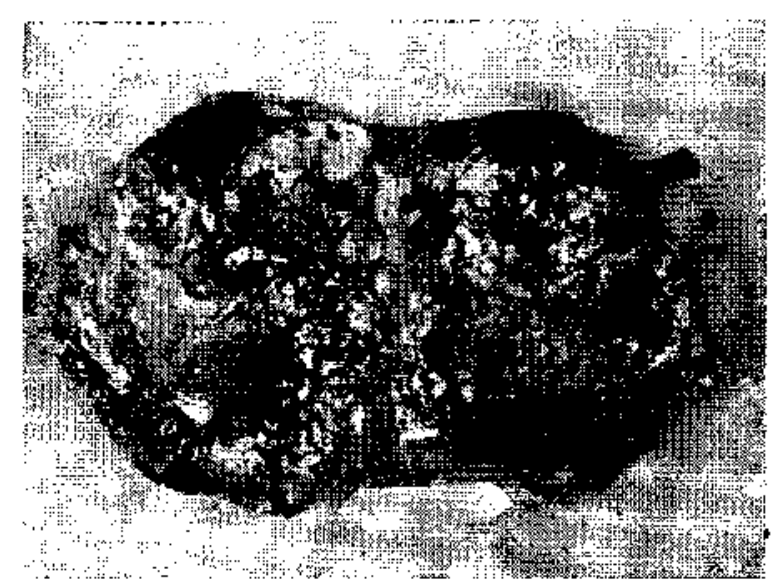

Figura No 5.- T de Wiims. Aspecto macroscopico

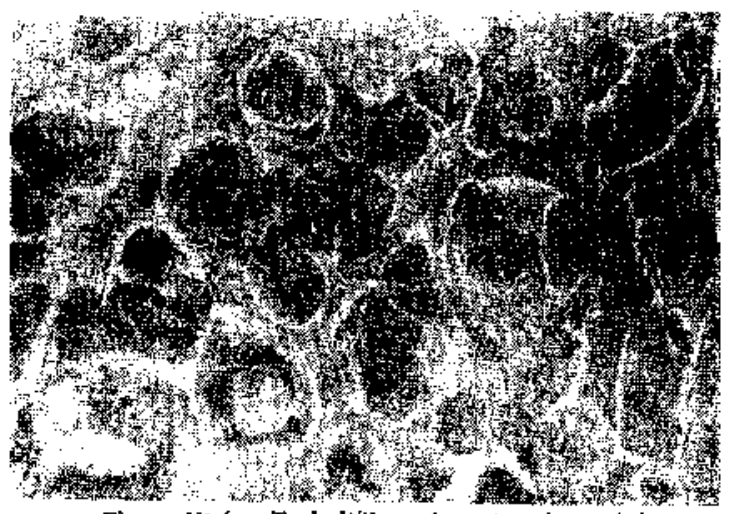

Figura No 6.- T, de Wilms. Aspecto microscópico.
Histológicamente lo que caracteriza al tumor de Wilms es la existencia de glomérulos y túbulos embrionarios o abortivos y un estroma constituido por células fusiformes mesenquimatosas que engloban túbulos, razón por la cual, se han considerado estos tipos de neoplasias como adenosarcomas. No es raro encontrar en el examen microscópico estructuras de músculo estriado o liso que es un signo auxiliar útil para establecer el diagnóstico definitivo. Con mucho menor frecuencia se pueden reconocer en este tipo de tumor, tejidos como: cartílago hialino, tejido conjuntivo fibroso, hueso, células adiposas y macrófagos cargados de lípidos. (Figura No 6).

En la tabla $N^{\circ} 7$ analizamos el tratamiento efectuado a nuestros enfermos, las complicaciones y resultados obtenidos.

a) Nefrectomía se practicó según indicaciones y técnica ya descrita en 15 casos, y en 9 de ellos se produjeron complicaciones. La ruptura del tumor y la del bazo ocurrieron en tumores de gran tamaño. Evisceración en 2 casos, 1 recibió radioterapia pre-operatoria y el otro, radioterapia muy precoz (a las $24 \mathrm{hrs}$.). Esto nos ha llevado a posponer la iniciación de la radioterapia hasta el $8^{\circ}$ día, fecha en que el estado de la herida operatoria nos da cierta seguridad de cicatrización.

b) Radioterapia se empleó en todos los pacientes y las dosis totales fluctuaron entre 2.000 y 5.000 rads. En 2 casos se usó en el pre-ope-

T A B L A N N 7

TRATAMIENTO DE 15 PACIENTES CON T. DE WILMS

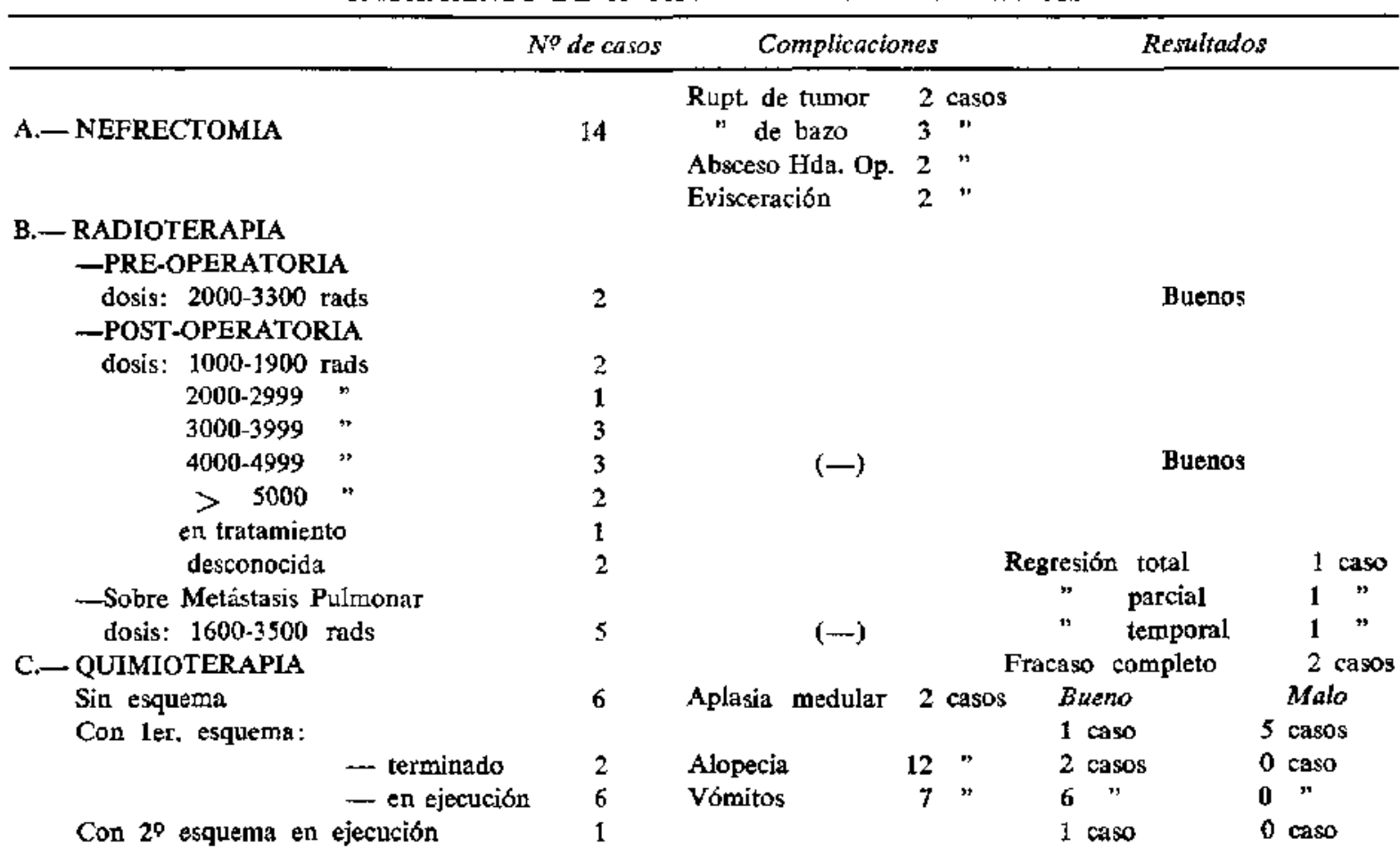


ratorio para reducir el tamaño del tumor y permitir la nefrectomía. EI uso de radioterapia preoperatoria no es la regla, ya que implica pérdida de tiempo que es valioso, y posibilidad de diseminación por inducir hiperemia, pero está indicada toda vez que exista gran riesgo quirúrgico dado el volumen del tumor.

En 14 casos se hizo radioterapia post-operatoria. En 2 pacientes se usó dosis menores que las aconsejadas (1.000 a $1.999 \mathrm{rads}$ ), en un niño en Estadio IV y Aplasia medular, y en otros que preBentaba infiltración intestinal. Dosis de 2.000 2.999 rads la recibió un solo caso previamente tratado con radioterapia pre-operatoria (3.300 rads). Ignoramos la dosis recibida en 2 pacientes en que el tratamiento se llevó a cabo en otros centros radioterapéuticos.

c) Quimioterapia. No se uś esquema en 6 pacientes, en uno que consultó antes de la iniciación del Centro de Tumores y en 5 por llegar en etapas muy avanzadas de la enfermedad. En 3 casos que presentaron metástasis pulmonares múltiples iniciales se usó quimioterapia múltiple (Actinornicina D, Ciclofosfamida, Vincristina y Prednisona) y lo mismo en 2 que bicieron metástasis pulmonares durante la evolución. Obtuvimos buen resultado en 1 caso y malo en 5 .

Con primer esquema terminado tenemos $2 \mathrm{pa}-$ cientes y 6 con esquema en ejecución. Con $2^{\circ}$ Esquema en ejecución un sola caso.

Las complicaciones que hemos observado en tratamiento quimioterápico son: aplasia medular ( 2 casos), alopecía (12 casos) y vómitos (7 casos).

\section{TABLA No 8 \\ ESTADIOS CLINICOS DE 15 PACIENTES CON TUMOR WILMS}

\begin{tabular}{ccc}
\hline Estadia & Inicial & Actual \\
\hline I & 5 & 4 \\
II & 0 & 0 \\
III & 5 & 4 \\
IV & 5 & 7 \\
& -5 & -15
\end{tabular}

En la Tabla No 8, de acuerdo a la clasificación de Fleming (10) hemos distribuido nuestros enfermos en los distintos estadios, en etapa inicial y actual de su enfermedad. De los 5 pertenecientes a Estadio I inicial, 4 continúan en ese mismo grado y están bien, $y$ uno pasó a grado IV por presentar metástasis pulmonar en su evolución.

De los 6 casos de grado III, uno solo hizo diseminación pulmonar y se lo reclasificó en estadio IV.

En grado IV inicial tuvimos 5 pacientes y 7 en la actualidad.
T A B L A No 9

PRONOSTICO EN RELACION A ESTADIO INICIAL

\begin{tabular}{cccc}
\hline & & \multicolumn{2}{c}{$N^{o}$ casos } \\
Estadio & No Total & Vivos & Fallecidos \\
\hline I & 5 & $5^{*}$ & 0 \\
II & 0 & 0 & - \\
III & 5 & 4 & 1 \\
IV & 5 & 2 & 3 \\
\cline { 3 - 4 } & & 11 & 4 \\
\hline
\end{tabular}

* 1 paciente vivo con enfermedad.

En el análisis de la tabla No 9 se observa que todos los niños que pertenecen a Estadio I están vivos y sin signos de recurrencia. El tiempo de sobrevida en los 5 casos es de: $7 \mathrm{~ms}$. $18 \mathrm{ds}$., $8 \mathrm{~ms}$. $23 \mathrm{ds}$, $10 \mathrm{~ms} .13 \mathrm{ds}$., 1 año $2 \mathrm{~ms}$, y 3 à $5 \mathrm{~ms}$. De Estadio III hay 4 niños vivos y aparentemente sin enfermedad, $y$ uno solo fallecido. El tiempo de sobrevida: 5 ms. 26 ds., 8 ms., 1 a. 1 ms., 1 a. $11 \mathrm{~ms}$., y 5 a. $8 \mathrm{~ms} .13 \mathrm{ds}$.

De 5 pacientes pertenecientes a Estadio IV hay 2 vivos y 3 fallecidos. Tiempo de sobrevida: $1 \mathrm{~ms}$. 12 ds., 4 ms. 21 ds., 8 ms., 9 ms. y 1 a. 6 ms.

TABLA No 10

RELACION ENTRE EDAD DE COMIENZO $Y$ SOBREVIDA

\begin{tabular}{lccccc}
\hline $\begin{array}{l}\text { Edad } \\
\text { (años) }\end{array}$ & Total & Vivos & Fallecidos & V/total & $\%$ \\
\hline$<2$ & 3 & 3 & 0 & $3 / 3$ & 100 \\
$2-4$ & 6 & 4 & 2 & $4 / 6$ & 60 \\
$>4$ & 6 & 4 & 2 & $4 / 6$ & 60
\end{tabular}

PRONOSTICO: Son factores decisivos:

- Edad: Todos los autores están de acuerdo en que el pronóstico es mejor en el niño menor de 2 años (10) (18) (27), hecho que vemos confirmado en nuestra casuística. También el T. congénito, que aparece en las primeras semanas de vida, es de curso benigno y no da metástasis (Tabla $N^{\circ} 10$ ).

- Estadio Clínico: Es mejor en el Estadio I, y el pronóstico se ensombrece a medida que aparece compromiso de estructuras vecinas y metástasis a distancia.

- Poder evolutivo del tumor: Collins postula un "Período de riesgo de recurrencia" que es igual a la edad del paciente en el momento del diagnóstico más 9 meses, y un "Período de curación" que es igual al doble de la edad más 9 meses (10) (18) (27). Para otros autores una sobrevida de 2 años libres de recurrencia 
serían suficientes para considerar curado un paciente (18) (27).

De nuestros casos 2 tienen sobrevida de más de 2 años (Estadio I y III inicial) y uno de ellos sobrepasó el tiempo de curación de Collins (Estadio III).

\section{RESUMEN}

Se estudian 15 pacientes con $T$. de Wilms retuidos en un periodo de 4 años 6 meses en el Centro de Tumores del Hospital Lais Calvo Mackenna.

- La mayor incidencia se observó an niños menores de 7 años.

- No hay fallecidos en el grupo de menores de 2 años de edad.

- Las dificultades en el diagnóstico precoz están dadas por el cuadro clínico inespecífico de este tumor.

- Se destaca el método de Fleming, que clasisica el $T$. de Wilms segtin Estadios clínicos y que actualmente utilizase en este Centro para manejo terapéutico y como índice pronóstico.

- Las metástasis pulmonares en estado inicial o durante la evolución son signos de mal pronóstico.

Resultados: II pacientes vivos y 4 fallecidos (Estadio $(V)$. 2 tienen sobrevida de más de 2 años (Estadio 1 y III) y uno de ellos (Estadio III) sobrepasó el tiempo de curación de Collins con una sobrevida de 5 años $8 \mathrm{~ms} .13 \mathrm{ds}$.

\section{SUMMARY}

15 patients with Wilms Tumor were seen on the last $4^{t / 2}$ years at the Tumors Departement of the Luis Calvo Mackenna Children's Hospital.

- Most of the cases appeared in patients under 7 years of age.

- No children died under 2 years.

- Diagnosis is difficult at the catly stages because of the lack of specific symptoms.

- Fleming's Classification was used to determine clinical stages and proved to be useful for the management und for proguosis purposes.

- Farly lung metastases suggest poor prognosis.

- 11 patients are alive. 2 bave more than 2 years of survival and one achieved Collins survival criteria. 4 patients died (IV Stage). 1968.

\section{Bibliografía}

1.-Ackerman L. and Del Regato J. Cancer Diagnosis, Treatment and Prognosis. St. Louis Mosby Co., 1970.

2.-Anderson E. et al. Bilateral Wilms'Tumor. Diagnosis and Management. Clin. Pediat. 7: 596-599,

3.-Bachman K. D. and Kroll. Der wilms tumor im ersten lebensjahr insbesondere uber 62 nephroblastome des neugeborenen. Dtsch. Med. Wschr. 94: 2598-2602, 1969.

4.- Balsaver A. M. et al. Ultrastructural Studies in Wilmstumors. Cancer 22: 417-427, 1968.

5.-Boxer L. and Smith D. Wilms'Tumor prior to onset of hemihypertrophy, Amer. J. Dis. Child. 120: $564-565,1970$.

6.-Chieh-Hsing Meng and Elkit N. Angiographic manifestations of Wilms'Tutnor. An abservation of six cases. Amer. J. Roentg. 15: 95-104, 1969.

7.-Dargeon $H$. Cancer in Childhood. St. Louis C. V. Mosby Co., 1940.

8.-Debergny P. et at. Tumeurs de Wilms chez l'enfant. Resultats therapeutiques d'une serie de 58 cas. Clin. Chir. Infant. et Orthop. 24: 98-109, 1969.

9.- Emparanza E. et al. Tumores Malignos en la Infancia. Rev. Chil. Ped. 39: 307-313, 1968.

10. Fleming 1 . and Johitson $W$. Clinical and pathologic staging as a guide in the management of Wilms" tumor. Cancer 26: 660-665, 1970.

11.-Filler $R$. et al. Hepatic Lobectomy in Childhood effects of X- ray and Chemotherapy. J. Pediat. Surg. 4: 31-41, 1969.

12.-Franmeni J. and Glass A. Wilms'tumor and Congenital aniridia. JAMA. 206: 825-829, 1968.

13.- Greene $F$. A review of the historical development and results of chemotherapy of wilms'tumor. Southern Med, J. 63: 1405-1408, 1970.

14.-Haicken B. and Miller D. Simultaneous Occurrence of congenital aniridia, hamartoma and Wilms' tumor. J. Pediat. 78: 497-501, 1971.

15.-Kenny G. M. et al. Erythropoietin levels in Wilms' Tumor patients. J. Urol. 104: 758-761, 1970.

16.- Kunkter $F$. Tratumiento del Cúncer en la práctica clínica. Ed. Salvat S. A. Barcelona, 1962. 
17.- Ledlie et al. Natural history and treatment of 335 cases occurring in England and Wales 1962-6. Brit. Med. J. 4: 195-200, 1970.

18. - Lelong $M$. Actualités Pediatriques. Ed. Doin y Cía. 1961.

19.- Lester $W$. $M$. Surgical management of solid tumors in childhood. Amer. J. Roentg. 105 105-109, 1969.

20.-- Lynch $H$. and Green G. Wilms'Tumor and congegenital heart disease. Amer. J. Dis. Child. 115: 723727, 1968.

21.- Martin J. and Rickham P. Pulmonary metastases in Wilms'Tumour: Treatment and Prognosis, Arch. of Dis, in Child. 45: 805-807, 1970.

22.- Martin L and Reyes P. An evaluation of 10 years' experience with retroperitoneal lmph node dissection for Wilms'tumor, J. Pediat. Surg. 4: 683$687,1969$.

23. - Nelson W. Tratado de Pediatría. Ed. Salvat S. A. Barcelona, 1971.

24.-Olmedo $R$. Tumor de Wilms. Actualidades en Cancerología. Sociedad Chilena de Cancerología. Sociedad Chilena de Cancerología, 1965.

25.-Pochedty C. Wilms Tumor I Clinical Features and Tumor Biology. New York J. Med. 71: 1089-1093, 1971.
26. - Pochedly C. Wilms'tumor II Diagnosis and Treatment. New York J. Med. 71: 1205-1209, 1971.

27. Pochedly C. Wilms'tumor IIf Special Problems in Therapy. New York J. Med. 71: 1526-1529, 1971.

28.-Smith $J$, et al. Detection of a small virus in a cultivated human WilmsTumor. Cancer, 24: 527.531, 1969.

29. - Sutow $\boldsymbol{W}$. et al. Comparison of survival curvs, 1956 versus 1962 , in children with WilmsTumor and Neuroblastoma, Ped. 45: 800-811, 1970.

30.-- Urrizola a. et al. Tumores abdominales en la infancia. Rev. Chil. Ped. 40: 898-905, 1969.

31.-Wagget $J$. and Koop C. Wilms'Tumor: Pre-operative radiotherapy and chemotherapy in the manegement of massive tumors. Cancer. 26: 338-340, 1970.

32.-Wedemeyer P. et al. Resection of metastases in Wilms'tumor: A report of three cases cured pulmonary and hepatic metastases. Ped. 41: 446-451, 1968.

33.-Woodard $J$. and Levine $M$. Nephroblastoma (Wilms'Tumor) and congenital aniridia. J. Urol. 101: $140-143,1969$. 